\title{
Kernos
}

Revue internationale et pluridisciplinaire de religion grecque antique

$10 \mid 1997$

Varia

\section{O. WATTEL-DE-CROIZANT, Les mosaïques représentant le mythe d'Europe}

\section{Catherine Lecomte}

\section{OpenEdition \\ Journals}

Édition électronique

URL : http://journals.openedition.org/kernos/676

DOI : $10.4000 /$ kernos. 676

ISSN : 2034-7871

\section{Éditeur}

Centre international d'étude de la religion grecque antique

\section{Édition imprimée}

Date de publication : 1 janvier 1997

Pagination : 336-338

ISSN : 0776-3824

\section{Référence électronique}

Catherine Lecomte, "O. WATTEL-DE-CrolzANT, Les mosaïques représentant le mythe d'Europe », Kernos [En ligne], 10 | 1997, mis en ligne le 12 avril 2011, consulté le 22 septembre 2020. URL : http:// journals.openedition.org/kernos/676 ; DOI : https://doi.org/10.4000/kernos.676 
duquel l'initié va rejoindre la divinité, Perséphone ou Dionysos. L'A., par son approche résolument originale des sources, renouvelle la perception de l'imaginaire funéraire aux époques archaïque et classique. Cette étude, à la fois iconographique et sociologique, constitue une riche contrepartie à des recherches archéologiques telles que celles entreprises par $\mathrm{J}$. de la Genière sur les nécropoles d'Italie du Sud ${ }^{33}$. L'ouvrage est accompagné d'illustrations bien choisies. Bibliographie et index des sources iconographiques en fin de volume.

Isabelle TASSIGNON (Université de Liège)

Odile WATTEL-DE CROIzANT, Les mosä̈ques représentant le mytbe d'Europe ( ${ }^{\text {er }}-V I^{e}$ siècles). Évolution et interprétation des modèles grecs en milieu romain, Paris, de Boccard, 1995. 1 vol. $16 \times 24 \mathrm{~cm}, 313$ p., 25 fig., 31 pl. (De l'archéologie à l'bistoire). ISBN : 2-7118-0091-9.

Madame Odile Wattel-de Croizant s'est proposé d'étudier dans cet ouvrage issu d'un doctorat d'État présenté à Tours en 1993 un ensemble de quarante-quatre mosaïques illustrant l'enlèvement d'Europe. Elle décrit la plupart des mosaïques, avec le vocabulaire technique propre à cet art, et les remet, aussi souvent qu'il est possible, dans leur contexte archéologique; enfin, elle joint, à la fin du volume, les reproductions en couleurs de nombre d'entre elles.

Le lecteur qui est initié à la science musivale (relative aux mosaïques) et qui possède des connaissances générales en archéologie sera sans conteste intéressé par cet impressionnant travail d'historienne de l'art, et ce n'est pas sur ce terrain que je porterai mes critiques. Je livrerai simplement quelques réflexions que la lecture de cet ouvrage peut susciter chez un philologue soucieux de comprendre les textes que l'A. invoque.

Dans un tel ouvrage, de toute évidence, il est impossible de se limiter à une description purement objective et matérielle: on en vient naturellement à une forme ou l'autre d'interprétation, sur la base de textes antiques cités en parallèle. Et c'est ici que les problèmes se posent. Il existe indubitablement, dans les représentations sur mosaïque, des couples formés d'un taureau à queue de Triton chevauché par une figure féminine. Cette femme est-elle une Europe ? Oui, dit Mme Wattel, en se référant à un passage des Dionysiaques de Nonnos le Panopolitain (I, 72-110). Que découvre celui qui se rapporte au texte grec? Que le taureau dont parle Nonnos n'est nullement un taureau marin par sa nature, mais qu'il est tout simplement comparé à un dauphin. C'est ainsi que la «queue de poisson » dont parle à plusieurs reprises Mme Wattel ne peut en aucune manière être celle du taureau qui porte Europe, mais celle d'un dauphin. Donc le texte de Nonnos ne peut servir à interpréter comme une Europe un personnage féminin chevauchant un animal hybride. Ceci disqualifie un certain nombre de représentations où l'A. interprète comme une Europe une femme à cheval sur le dos d'un taureau à queue de poisson.

Mme Wattel semble considérer qu'Europe est une « déesse au taureau », expression qu'elle emploie à de très nombreuses reprises. Mais elle n'avance aucune preuve,

33 J. DE LA GENIÈRE, Les sociêtés antiques à travers leurs nécropoles, in MEFRA, 102 (1990), p. 8491. 
ni tirée des textes, ni tirée des représentations figurées. On est forcé de la croire sur parole. Dans cet ordre d'idée, on aurait aimé que l'A. pose les questions suivantes et y réponde : Europe est-elle une déesse? Est-elle une ancienne déesse dévaluée ? Est-elle une héroïne? A-t-elle hérité son iconographie d'une « déesse au taureau » et, si oui, laquelle?

À propos des homonymes de l'Europe "phénicienne », l'A. mentionne une Déméter-Europe dont le culte serait célébré à Lébadée et à Sicyone. Le premier culte est bien connu. Pour le deuxième, l'A. ne cite pas ses sources, mais ça ne peut être que Pausanias (II, 5, 8 et II, 11, 2). Mais, dans le texte de Pausanias, s'il est bien question de Déméter, le nom d'Europe n'apparaît pas; c'est Déméter seule qui nourrit le fils du roi Plemnaios (et pas "Plemaios", comme l'écrit chaque fois l'A.). Toujours à propos des homonymes d'Europe, Hégésippe et Conon feraient de Cadmos l'époux d'une souveraine dénommée Europe. Mais si l'on se rapporte au texte, fragmentaire, d'Hégésippe, on lit que Cadmos, parti à la recherche d'Europe en compagnie de la mère de celle-ci, Téléphaé, arrive en Thrace où règne une souveraine dénommée elle aussi Europe, mais qui n'a rien à voir avec celle qu'il cherche. On ne trouve pas davantage de mariage entre Cadmos et une Europe chez Conon (dans la référence «Conon, 32 » que donne l'A., il faut comprendre Conon, 26 F 1, XXXII Jacoby).

Voici quelques autres critiques de détail.

Pour justifier la présence d'Europe sur une métope de Sélinunte (sic), l'A. tente, sans y parvenir, de déceler des influences crétoises dans cette ville et affirme, sans aucune preuve à l'appui, que « la diffusion de cette légende à Sélinunte pourrait (...) s'expliquer par la survivance d'une tradition orale qui aurait conservé le souvenir d'une Crétoise en ces lieux, dans la période qui précéda la colonisation ». Trois remarques à ce sujet. D'abord, il faudrait savoir si Europe est crétoise, phénicienne ou béotienne, adjectifs que, dans le cours de son livre, l'A. applique à l'héroïne, avant de chercher à expliquer pourquoi un tel sujet a été choisi pour décorer une métope. Ensuite, il aurait fallu étudier l'ensemble des métopes, voire du temple et du site, avant de tenter de trouver désespérément, en raison d'une seule représentation, une présence crétoise à Sélinonte. Enfin, cette présence crétoise est inutile si vraiment, comme l'écrit l'A., « les représentations du mythe d'Europe devinrent un motif très en vogue aux alentours des années 600 et un thème littéraire panhellénique à partir du $\mathrm{VI}^{\mathrm{e}}$ siècle, »

Il existe une abondante bibliographie bien plus récente (1863 !) que celle donnée par l'A. sur la peinture de l'enlèvement d'Europe chez Achille Tatius et sur son rôle dans le roman. Voir par exemple T. HÄGG, Narrative Technique in the Ancient Greek Romances. Studies of Chariton, Xenophon Ephesius, and Acbilles Tatius, Stockholm, 1971 (Acta Instituti Atheniensis Regni Sueciae, Series in-8, VIII) et Shadi BarTSCH, Decoding the Ancient Novel. The Reader and the Role of Description in Heliodorus and Acbilles Tatius, Princeton University Press, 1989. Chez Moschos, ce n'est pas un simple taureau envoyé par Zeus qui apparaît dans la prairie où Europe cueille des fleurs, mais c'est le dieu lui-même métamorphosé en taureau (Moschos, Europe, 79: ý(veTo taũpos). Donc, l'étude que Mme Wattel consacre à cette prétendue innovation, à savoir la métamorphose de Jupiter, qu'Ovide aurait introduite en modifiant la tradition rapportée par Moschos, est sans objet.

Mme Wattel présente la figure 17 de son ouvrage comme le groupe statuaire de bronze, dû au sculpteur Pythagoras, qui se trouvait à Tarente. Or, ce que reproduit cette illustration est en réalité un groupe de marbre - et non de bronze - du thêâtre de 
Gortyne en Crète - et non en Grande-Grèce - daté du I $\mathrm{I}^{\mathrm{e}}$ ou du II ${ }^{\mathrm{e}}$ siècle de notre ère - il est donc postérieur d'au moins six siècles à Pythagoras. Une note à la fin du chapitre, il est vrai, suggère que le groupe de marbre « pourrait (...) être la copie » du groupe de bronze de Pythagoras. Dès lors, une hypothèse formulée en note devient une certitude dans le corps du texte.

À moins que Virgile n'ait écrit l'Énéide en distiques élégiaques, on voit mal comment les vers Invida si Tauri vidisset Juno natatus / Iustius Aeolias isset ad usque domos pourraient être tirés de ce poème, comme l'écrit Mme Wattel. Ces vers, inscrits sur la mosaïque de Lullingstone, sont simplement une allusion au début de l'Énéide (I, 50-53) et veulent dire que si la jalouse Junon avait vu nager le taureau, elle aurait eu de bonnes raisons d'aller à la demeure d'Éole, des raisons meilleures encore que celles qui la faisaient poursuivre les Troyens.

Et l'on pourrait citer d'autres exemples de ce genre. En définitive, pour interpréter les représentations figurées, qui font allusion à des traditions religieuses et littéraires, il est indispensable de lire et d'étudier correctement les textes.

Catherine LECOMTE

(Université de Liège)

Stella Georgoudr, Jean-Pierre Vernant (éds), Mythes grecs au figuré, de l'antiquité au baroque, Paris, Gallimard, 1996. 1 vol. $16,5 \times 21 \mathrm{~cm}, 234$ p., 58 ill. (Coll. Le temps des images). ISBN : 2-07-73910-4.

Depuis quelques années déjà, le mythe grec fait l'objet de discussions passionnées et passionnantes, dont Jean-Pierre VERNANT fait état dans un chapitre introductif sur Les frontières du mythe. Mais ce sont plus particulièrement les rapports entre les récits mythiques et leur transposition figurative, d'une part, et le cheminement de certains mythes qui se détachent de leur origine pour se donner de « nouveaux habits », d'autre part, qu'illustrent ces quelques "échantillons » issus d'un colloque tenu à Delphes en 1993 sur La mythologie grecque et l'art baroque.

Puisque, décidément, la Grèce est au centre dès qu'on parle de « mythe », c'est en Grèce que commence ce parcours tout en questions; car dès le $\mathrm{v}^{\mathrm{e}}$ siècle av. J.-C., on interroge les mythes : "façons de dire traditionnelles et non fiables » pour Platon, fabulations invérifiables pour Thucydide, mais aussi histoires fondatrices qu'il convient d'harmoniser et d'articuler entre elles pour les logographes, les mythes deviennent peu à peu, - surtout avec l'époque impériale et la mise sous tutelle d'une Grèce en quête de sa nouvelle identité, - une mythologie, un savoir spécifique, un champ d'étude particulier, délié de la littérature, séparé de l'histoire et à l'écart des enjeux idéologiques traditionnels. Ils sont alors prêts pour les transformations et les resémantisations des siècles suivants (J.-P. VernANT). Avant d'envisager, avec Danaé (Fr. LisSARAGUE) et Andromède (Fr. Frontisi-DucrouX), ce que sont certaines de ces transformations, la Grèce nous retient encore avec ce curieux «panthéon condensé » que forment les "Douze dieux » (St. GeORGOUdi). Sur le terrain des pratiques, des narrations mythiques et des représentations figurées, les idées reçues à leur sujet depuis l'article d'O. WEINREICH dans le Lexicon de ROSCHER sont systématiquement mises en cause, avec une grande force de conviction : il est tout aussi vain d'en chercher l'origine en Ionie que d'en établir un prétendu "canon », en fait introuvable. 